University of New Hampshire

University of New Hampshire Scholars' Repository

Space Science Center

Institute for the Study of Earth, Oceans, and

Space (EOS)

9-22-2007

\title{
Using LaX scintillator in a new low-background Compton telescope
}

James M. Ryan

University of New Hampshire, James.Ryan@unh.edu

Peter F. Bloser

University of New Hampshire, Peter.Bloser@unh.edu

John R. Macri

University of New Hampshire - Main Campus, John.Macri@unh.edu

Mark L. McConnell

University of New Hampshire - Main Campus, mark.mcconnell@unh.edu

Follow this and additional works at: https://scholars.unh.edu/ssc

Part of the Astrophysics and Astronomy Commons

\section{Recommended Citation}

James M. Ryan ; Peter F. Bloser ; John R. Macri and Mark L. McConnell "Using LaX scintillator in a new low-background Compton telescope", Proc. SPIE 6707, Penetrating Radiation Systems and Applications VIII, 670703 (September 22, 2007); doi:10.1117/12.740646; http://dx.doi.org/10.1117/12.740646

This Conference Proceeding is brought to you for free and open access by the Institute for the Study of Earth, Oceans, and Space (EOS) at University of New Hampshire Scholars' Repository. It has been accepted for inclusion in Space Science Center by an authorized administrator of University of New Hampshire Scholars' Repository. For more information, please contact Scholarly.Communication@unh.edu. 


\title{
Using LaX scintillator in a new low-background Compton telescope
}

\author{
James M. Ryan, Peter F. Bloser, John R. Macri, Mark L. McConnell \\ Space Science Center, University of New Hampshire, Durham, NH 03824
}

\begin{abstract}
The ability of Compton telescopes to perform imaging and spectroscopy in space depends directly on the speed and energy resolution of the calorimeter detectors in the telescope. The calorimeter detectors flown on space-borne or balloon-borne Compton telescopes have included $\mathrm{NaI}(\mathrm{Tl})$, CsI(Na), HPGe and liquid organic scintillator. By employing LaX scintillators for the calorimeter, one can take advantage of the unique speed and resolving power of the material to improve the instrument sensitivity and simultaneously enhance its spectroscopic performance and thus its imaging performance. We present a concept for a space-borne Compton telescope that employs LaX as a calorimeter and estimate the improvement in sensitivity over past realizations of Compton telescopes. With some preliminary laboratory measurements, we estimate that in key energy bands, typically corrupted with neutron-induced internal nuclear emissions, this design enjoys a twenty-fold improvement in background rejection.
\end{abstract}

Keywords: $\mathrm{LaX}$ scintillators, $\mathrm{LaBr}_{3}$, Compton telescopes, instrumentation

\section{INTRODUCTION}

Compton telescopes are the instruments of choice for spectroscopic imaging of the sky in the difficult energy range of $400 \mathrm{keV}$ to $10 \mathrm{MeV}$ (Ryan 2004). This is mainly so because the coincidence requirement greatly reduces the intrusion of single photon and single-particle radioactive decays and scatters into the data. The intrinsic directionality of Compton telescopes further reduces background. The "classical" Compton telescope, COMPTEL (Schönfelder et al. 1993), employed two detecting arrays separated by $1.6 \mathrm{~m}$, with a good event being one with near simultaneous interactions in the two detectors. Compton scatters in the forward detector D1 were measured for location, energy deposit and pulse shape. D1 was composed of liquid organic scintillator. Location and energy deposit were measured for scatters into the rearward detector D2 and the time-of-flight (ToF) was measured between the detector triggers. D2 was composed of $\mathrm{NaI}(\mathrm{Tl})$. From the positions and energy deposits in the two detectors, the source of the incident $\gamma$-ray photon could be constrained to lie on an "event circle" on the sky using the Compton-scatter formula. The coincidence requirement greatly reduced the effective area of the instrument, but reduced the background even further, yielding a net improvement in sensitivity over single-detector systems. COMPTEL produced the first and only map of ${ }^{26} \mathrm{Al}$ in the galaxy, discovered AGN emission in the $\mathrm{MeV}$ range, measured prolonged $\gamma$-ray emission from solar flares, and much more, including a measure of the cosmic diffuse flux in the 1-30 MeV range.

A broad range of astrophysical science is best studied via observations in this so-called medium-energy $\gamma$-ray band, from roughly $0.4-10 \mathrm{MeV}$. Medium-energy $\gamma$ rays probe extreme physical conditions in the Universe that give rise to nuclear interactions and relativistic particles. The physics at work determines how stars die and new elements are born. Investigating this radiation will help us understand the behavior of black holes and the fate of the Universe. The only Compton telescope in this energy range to fly in space with sufficient sensitivity to make useful astronomical observations was COMPTEL, which flew on the CGRO satellite from 1991-2000. COMPTEL provided only a dim, blurry view of the $\mathrm{MeV} \gamma$-ray Universe, but it was sufficient to demonstrate that the science return of a more sensitive instrument will be rich. Specifically, a significantly more sensitive instrument would address the following:

- Search for and measure supernova (SN) emissions to better understand the dynamics of the explosion process and the creation of the elements. The anomalous redshift velocities of distant Type Ia SNe have led to a critical review of the cosmological constant. It is important to fully understand the Type Ia process because it is used now as a standard candle to evaluate the Hubble constant. COMPTEL was able to place upper limits on ${ }^{56} \mathrm{Co} \gamma$ ray line emission from one SN and marginally measure another (Morris et al. 1998). With a 10× improvement in sensitivity, it is likely that a Type Ia SN will occur in a five-year period within a distance where good measurements can be conducted to test different models.

- Measure the radioactive decay line emission from supernova remnants (SNR). Improving upon the ${ }^{44} \mathrm{Ti}$ luminosity measurements of Iyudin et al. (1994) of Cas-A would be accompanied by luminosity measurements

Penetrating Radiation Systems and Applications VIII, edited by F. Patrick Doty, H. Bradford Barber, Hans Roehrig

Proc. of SPIE Vol. 6707, 670703, (2007) -0277-786X/07/\$18 - doi: 10.1117/12.740646

Proc. of SPIE Vol. 6707 670703-1 
of ${ }^{26} \mathrm{Al}$ and ${ }^{60} \mathrm{Fe}$. These isotopes drive the later phases of the supernova expansion and populate the Galaxy with radioactive ash.

- The isotope ${ }^{26} \mathrm{Al}$ populates the plane of the Galaxy producing a diffuse Galactic emission. It was mapped by Diehl et al. (1995), but higher definition versions of these maps are necessary to properly associate the emission with distinct galactic structures and their progenitor stars.

- The origin of the diffuse Galactic continuum emission at $\mathrm{MeV}$ energies is still uncertain: current models of galactic $\gamma$-ray production in cosmic ray/gas interactions fail to provide a satisfactory explanation (Strong et al. 2000) for this galactic glow.

- Black-hole candidates and AGNs exhibit emission in the MeV range, probably from the re-energization of X rays by scattering off high-energy electrons. The MeV tail on the spectrum of Cyg X-1 (McConnell et al. 2002) represents the kind of exciting physics that can emerge when studying such objects. AGNs have been associated with emission above $100 \mathrm{MeV}$, but this is likely due to the limited sensitivity in the $\mathrm{MeV}$ band. A broader picture of AGNs will probably emerge when more are measured in the MeV band. The object 3C273 is such an example of an AGN that is most luminous in $\mathrm{MeV} \gamma$ rays.

- Classical novae produce isotopes that radiate in the MeV range. Measurements of these isotopic abundances will constrain the dynamics of the origin of lighter elements.

- Pulsars should be common in the Galaxy if one has the sensitivity to detect them. COMPTEL detected a handful of them, all displaying different behaviors in spectrum, light curve and frequency. This diversity makes categorizing them difficult. A larger set of $\gamma$-ray pulsars might exhibit trends that could be used to understand them.

- $\gamma$-ray bursts exhibit some of their most interesting features at energies higher than the classical 100-200 keV. They have persisted for time scales of an hour at GeV energies (Dingus et al. 1995), but studies of their longterm behavior at $\mathrm{MeV}$ energies have been precluded by the sensitivity of $\mathrm{MeV}$ instruments.

- The spectrum of the cosmic diffuse $\gamma(\mathrm{CDG})$ emission appears from COMPTEL, EGRET and SMM data to be comprised of several components. These components include active galaxies and supernovae. The supernova component is the least resolved and understood. A new mission would greatly improve on the COMPTEL measurements of this spectrum from below $1 \mathrm{MeV}$ to $10 \mathrm{MeV}$ and help establish the magnitudes of the different contributions.

- Solar Flares exhibit the most complex spectra in the sky. The richness of nuclear lines, bremsstrahlung emission, positron annihilation, and neutron-capture contains much information about the processes of ion acceleration that produces these lines. Because of the complexity of the spectrum, the diagonal instrument response of a Compton telescope will greatly aid in the interpretation of the data.

- Many of the phenomena described above probably produce $\gamma$ radiation that is at least partially polarized. A $\gamma$-ray telescope based on Compton scattering is in principle sensitive to polarization and thus offers a new diagnostic tool for the study of high-energy astrophysical processes (McConnell \& Ryan 2004).

To accomplish these scientific goals a new telescope design superior to that of COMPTEL is necessary.

\section{TECHNICAL ISSUES}

Scintillator-based Compton telescopes have flown on balloons as far back as the late 1960s (e.g., Schönfelder et al. 1977; White et al. 1977; Ryan 1978). All achieved success, to different degrees, in conducting astrophysical measurements, from $\mathrm{MeV}$ measurements of secondary neutral cosmic radiation to measurements of the Crab and Vela pulsars and the Crab nebula to mapping, albeit crude, of the ${ }^{26} \mathrm{Al}$ galactic radiation to the cosmic diffuse flux. But with the COMPTEL experiment this basic design ran up against the limits of background that one experiences on a space platform. In the end, it was our inability to deal with the COMPTEL background rate, of order $4 \times$ above early estimates, that limited the scientific return from the mission. In fact, in the most problematic energy ranges, it was the uncontrolled background fluctuations rather than photon statistics that limited the instrument sensitivity that limited the instrument sensitivity(e.g., Bloemen et al., 1999). However, on the bright side, the instrumental background was exhaustively studied and cataloged as part of the measurement of the $\mathrm{MeV}$ cosmic diffuse background. Without introducing new channels of background, steps can now be taken to mitigate all major components of the instrumental background that plagued COMPTEL, enabling a re-design that can achieve far greater sensitivity. 
Briefly, COMPTEL operated in the following way as illustrated in Fig. 1. A $\gamma$ ray scatters in D1, the upper liquid scintillator assembly. The liquid scintillator was less than one $\gamma$ mean free path thick. The scattered $\gamma$ ray would then ideally be fully absorbed in $\mathrm{D} 2$, the calorimeter $\mathrm{NaI}$ assembly. The locations of the interactions are determined from relative pulse heights and the energy measurements from the summed pulse heights. These measurements restrict the incident $\gamma$ direction to a circle on the celestial sphere. The width and radius of the circle (actually an annulus) are fixed by the accuracy of the position locations and the accuracy of the energy measurements. Through one of several possible

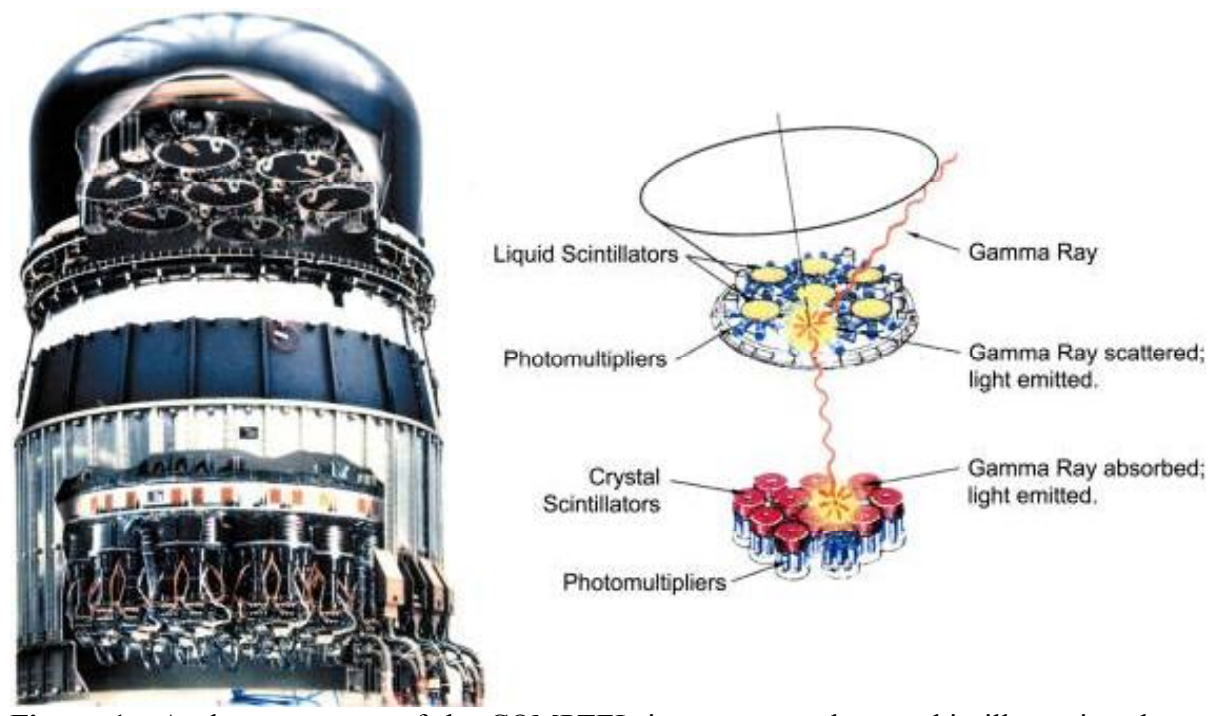

Figure 1. A photo montage of the COMPTEL instrument and a graphic illustrating the method of detection, where $\gamma$ rays scatter in the upper detector assembly (D1) and are absorbed in the lower (calorimeter) detector assembly (D2) with the resulting restriction on the incident photon direction.

algorithms, the statistical ensemble of many such circles provided the location of a point source. The events consistent with a source direction also provide its spectrum. The background that one fights in analyzing a source primarily comes from activation and neutron-induced photons within the spacecraft or instrument and chance coincidences between independent signals in the two detector assemblies. Charged particles are effectively eliminated with plastic charged particle shields.

Below we describe the historical methods, both in hardware and analysis, for excluding background in the COMPTEL experiment. We then describe, in some detail, (1) the nature of the COMPTEL background, and (2) new methods in hardware that can mitigate the effect of these sources of instrumental background. Lastly, we present a rough projection of the sensitivity that one could reasonably expect from an Explorer version of such an instrument.

The hardware methods for reducing background in COMPTEL were (1) charged particle shields to exclude effects of proton and electron induced prompt $\gamma$ rays, (2) time-of-flight with a final (after processing) 4-ns coincidence window that restricted events to forward directions and eliminated single triggers for photons and radioactive decays, and (3) pulse-shape-discrimination that reduced the effect of inelastic neutron scatters off carbon in the organic scintillators.

In the data analysis and processing, we selected events in the forward ToF interval, the electron-pulse-shape interval and relative energy deposits in D1 and D2 that corresponded to Compton scatters typically less than $36^{\circ}$. Finally, the resulting event circle for each event was not allowed to come closer to the horizon than $10^{\circ}$. These losses of solid angle significantly reduced the effective area of the instrument. The remaining events constituted the highest quality events that were then fed into imaging and spectroscopic algorithms. The hardware and on-board software restrictions reduced the on-board count rate (in deep space observations) to $\sim 10 \mathrm{~Hz}$ from 0.75 to $30 \mathrm{MeV}$. Ground software data cuts further reduced the count rate to $\sim 5 \mathrm{~Hz}$. This should be compared to the expected cosmic diffuse true event rate of $\sim 1 \mathrm{~Hz}$, i.e., the dominant contributor to the real $\gamma$ count rate.

\subsection{The Nature of the COMPTEL Background}

One of the goals of instruments such as this has always been to accurately measure the so-called $\mathrm{MeV}$ cosmic diffuse background flux, an unresolved but intense cosmic radiation field of uncertain origin. Because no structure has been observed and little expected in this energy range, imaging is of little value. Rather, to measure the spectrum, all steps 
must be taken to minimize the background to maximize the signal-to-noise ratio. A major loss of effective area could always be made up with long exposures.

In the course of these investigations with COMPTEL (Kappadath 1998; Weidenspointner 1999; Weidenspointner et al. 2001), much was learned about the nature of the Compton telescope background, i.e., the limiting factor in these measurements. We learned that although the sensitivity to single photon backgrounds, such as that from radioactive ${ }^{40} \mathrm{~K}$ contamination in photomultiplier tube glass, was largely eliminated by the double Compton technique, prompt doublephoton background produced in the instrument and single photon background production in the detector elements themselves proved to be the limiting factors for the instrument sensitivity.

Specifically, ${ }^{22} \mathrm{Na}$ emission from activation in the $\mathrm{D} 2$ detector (NaI based) was never detected in the main data stream even though its production is well documented in the GRS of SMM (Share et al. 1988) and in OSSE on the Compton Observatory. Gamma rays from ${ }^{22} \mathrm{Na}$ decays could easily be rejected because they had a unique ToF signature, i.e., they traveled in the wrong direction. However, photons from ${ }^{24} \mathrm{Na}$ and ${ }^{22} \mathrm{Na}$ produced in the activated aluminum of the D1 detector were a persistent, time variable and elusive background affecting all measurements below $4 \mathrm{MeV}$ (Bloemen et al. 1999). ${ }^{24} \mathrm{Na}$ was a problem because the isotope emits two simultaneous photons of 1.37 and $2.75 \mathrm{MeV}$ with one triggering D1 and the other triggering D2. The ToF signature was almost exactly what one expects from a cosmic $\gamma$ ray. However, upon close inspection slight distortions were seen in the ToF spectrum of otherwise good photons. The distortions were in the direction of slightly smaller ToF values than normal, indicating that the second photon of the $24 \mathrm{Na}$ cascade was part way to D2 when the other photon triggered D1.

The other major problem was that the organic scintillators used in D1 and the charged particle shield thermalized and captured free neutrons produced by cosmic ray interactions in the instrument, spacecraft or Earth's atmosphere. The result was a strong neutron-capture $2.223 \mathrm{MeV}$ emission line in the aperture of the instrument. These photons properly scattered and could not be distinguished from otherwise good cosmic photons. This adversely affected all measurements at and below $2.2 \mathrm{MeV}$.

These two effects can be seen in the COMPTEL mean-ToF plot as a function of measured $\gamma$-ray energy in Fig. 2. Plotted in Fig. 2 is the centroid of the ToF peak for the best-selected events in the COMPTEL data stream. The ToF channel of a good cosmic $\gamma$ ray for COMPTEL was, by design, 120. As evident in Fig. 2, this was satisfied for energies of $2.2 \mathrm{MeV}$ and above $9 \mathrm{MeV}$. At all other energies the quadratic fit (deemed best) was always below 120 indicating that double-photon cascade background was contaminating the data. The ToF centroid was correct at $2.2 \mathrm{MeV}$ because those photons were intense and true double scatters, while above $9 \mathrm{MeV}$ there are no nuclear states to provide a doublephoton cascade. For other energies, the offset of the centroid is more than $500 \mathrm{ps}$ from the proper mean-ToF value. This can be understood because a single D1 detector cell had a radius of $14 \mathrm{~cm}$ or $\sim 500$ light-ps. Cascades initiating in the housing or platform of a D1 cell would typically travel for $500 \mathrm{ps}$ before scattering in a D1 cell. In this time the other photon of the cascade was $14 \mathrm{~cm}$ on its way toward D2, thereby registering as a coincidence $\sim 500 \mathrm{ps}$ earlier than the proper ToF. More detailed analyses of the ToF spectrum by Kappadath (1998) and Weidenspointner (1999) resolved this double-photon cascade component, placing it almost a full $1 \mathrm{~ns}$ away from the proper cosmic-photon value. COMPTEL with its 1.5 -ns ToF resolution had a difficult time dealing with this background component since the two ToF distributions merged into a Gaussian-looking feature. However, one can now imagine a Compton telescope with a ToF resolution of $\sim 500 \mathrm{ps}$ or better neatly resolving the cascade background from the cosmic flux-meaning that only cascades initiating a few $\mathrm{cm}$ or less from a D1 cell would contaminate the data.

Cosmic-ray interactions in the instrument, spacecraft or the nearby Earth's atmosphere produce an intense neutron radiation field. The neutrons would often thermalize in and around the D1 detector. Once inside a D1 cell they would combine with hydrogen to form deuterium, releasing the deuteron binding energy in a single photon with energy of $2.223 \mathrm{MeV}$ that could, in turn, double scatter and enter the data stream. Organic scintillators with similar properties to those used in COMPTEL are available with hydrogen replaced by deuterium. A Compton telescope using deuterated organic scintillators (in D1 and in the surrounding charged particle shields) would be immune from this form of background.

The major background events types in COMPTEL are illustrated in Fig. 3 (Kappadath 1998). Sub-ns ToF resolution measurements can eliminate all the event types shown in Fig. 3 except Type A and a small fraction of Types B \& C. Type A events include (1) neutron captures on hydrogen in the organic scintillator of D1, (2) neutron capture on 


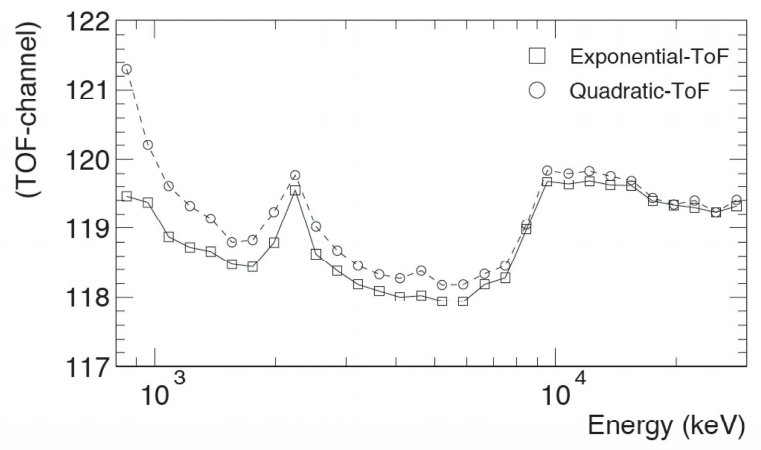

Figure 2: Centroid of the COMPEL ToF peak as a function of energy.

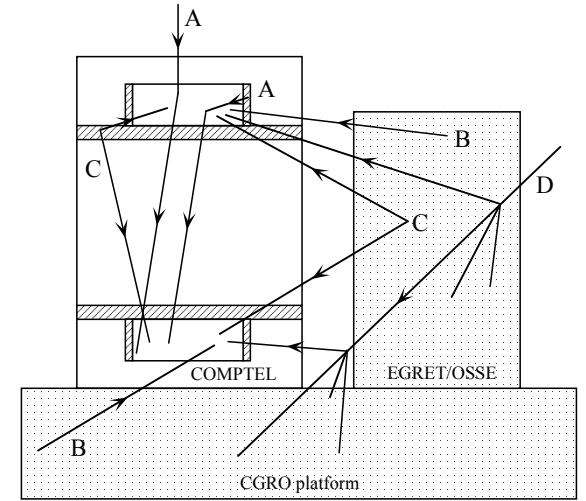

Figure 3: The major types of background event in COMPTEL (see text).

hydrogen in the organic scintillator of the forward charged particle shield, (3) single photon penetration of a D1 cell (cosmic $\gamma$ rays) and (4) photons from inelastic neutron-carbon scatters with the carbon decaying to the ground level. Lastly, activation of the carbon in the organic scintillator of D1 can produce events. In particular ${ }^{91} \mathrm{C}$ emits a $\beta^{+}$particle in D1 with a subsequent $511 \mathrm{keV}$ photon triggering D2. Type B events inside the ToF window and Type C events occurring close to D1 are also not eliminated entirely. Many of these events can, however, be eliminated by restricting the computed scatter angle. For example, most of the ${ }^{11} \mathrm{C}$ events in COMPTEL were eliminated because of the large positron energy deposits in D1 relative to the energy deposited in D2, implying a large Compton scatter if it were a cosmic $\gamma$ ray. Similarly, ${ }^{40} \mathrm{~K}$ photons from the glass of the photomultiplier tubes (PMTs) must undergo a large scatter in D1 to make it to D2.

Several lessons can be learned from the COMPTEL experience. These include (1) the ToF resolution must be as small as possible. This reduces the frequency of Type B events and reduces the susceptible volume of Type $\mathrm{C}$ events to that very close to D1. For example, shown in Fig. 8 (adapted from Kappadath 1998) is the highly selected ToF spectrum from 4.2 to $6 \mathrm{MeV}$ used in the CDG analysis (see below). Shown are the fitted Gaussian curves for the Type A events and Type $\mathrm{C}$ events. Fig. 9 shows the expected ToF curves from an instrument with a 0.4-ns ToF resolution. The components are now resolvable. New instrument geometries can be envisioned and evaluated to ensure that these components remain resolvable, while optimizing other instrument parameters, such as efficiency. A side benefit of a small ToF resolution is that that the individual D1 and D2 cell volumes must be small, thereby increasing the light collection efficiency and improving energy resolution.

Lesson (2) is that the passive mass surrounding D1 (and only D1) be small and of a composition that resists activation into multi-photon decays. For example, a space-flight mission might use Be instead of Al. Finally, (3) eliminate hydrogen in D1 and the forward charged particle shield. This involves using deuterated organic scintillators.

The effect of carbon in D1 is not easily reduced, but indications are that this is not a major effect in any case. For example, no $4.43 \mathrm{MeV}$ line has ever been detected in the COMPTEL background, placing an upper limit on the intensity of this component.

To summarize: the actions that can greatly affect the background count rate without affecting efficiency are (1) reduce the ToF resolution with a goal of $500 \mathrm{ps}$ or better, (2) employ deuterated pulse-shape sensitive organic scintillators in strategic locations and (3) reduce the mass immediately surrounding D1, ideally with a composition resistant to activation. Below we describe the expected reduction in background rates for the various event types in one possible configuration.

\subsection{Current Status of Fast Scintillators}

The detector performance (fast timing, good energy resolution, good stopping power) needed to improve on the sensitivity of COMPTEL can now be realized with modern fast scintillator materials. One promising material that has been the subject of intense study in recent years is cerium-doped Lanthanum Bromide $\left(\mathrm{LaBr}_{3}: \mathrm{Ce}\right)$. Compared with "traditional" scintillators such as $\mathrm{NaI}(\mathrm{Tl})$ (used in COMPTEL D2), $\mathrm{LaBr}_{3}$ offers higher light output (60,000 vs. 49,000 photons $\mathrm{MeV}^{-1}$ ), higher density and stopping power (5.3 vs. $\left.3.7 \mathrm{~g}-\mathrm{cm}^{-3}\right)$, faster response (1/e decay time of $26 \mathrm{~ns}$ vs. 250 ns), and superior energy resolution (2.8\% vs. $7 \%$ FWHM at $662 \mathrm{keV})$ (Shah et al. 2003). A ToF resolution as short as 
260 ps (FWHM) has been measured between $\mathrm{LaBr}_{3}$ detectors and other fast scintillators (Shah et al. 2003; Kuhn et al. 2004).

\section{A DESIGN USING LAX SCINTILLATORS}

We have developed a baseline conceptual model of a modern Compton telescope utilizing $\mathrm{LaBr}_{3}$ in $\mathrm{D} 2$ for improved efficiency, timing, and energy resolution. A subsection of the model is shown in Fig. 4. The model comprises 2 layers of deuterated liquid scintillator for D1 and 2 layers of $\mathrm{LaBr}_{3}$ detectors for $\mathrm{D} 2$. Each layer is $100 \mathrm{~cm} \times 100 \mathrm{~cm}$, and the separation between D1 and D2 is $75 \mathrm{~cm}$. Each individual D1 or D2 detector element is a 2-cm cube. No finer spatial resolution is assumed because we wish to study the performance achievable with excellent time resolution and good

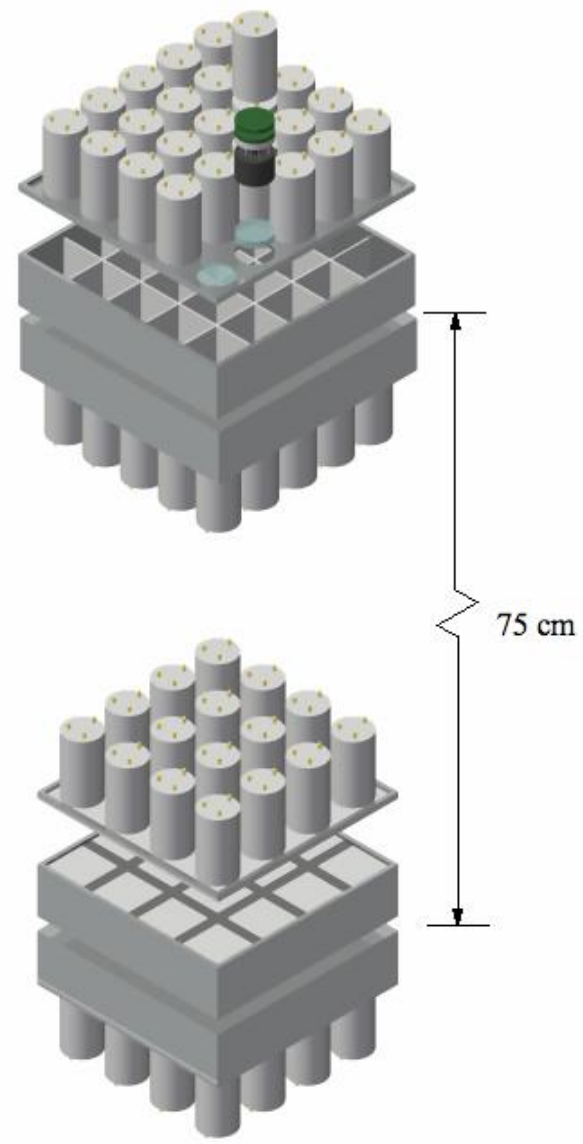

Figure 4. A $10 \mathrm{~cm} \times 10 \mathrm{~cm}$ subsection of the model Compton telescope using deuterated liquid scintillator for D1 and $\mathrm{LaBr}_{3}$ for D2. See text for details.

energy resolution while keeping the channel count modest ( $\sim 8000$ in the current mass model). For light readout a compact PMT (e.g., Hamamatsu R7400) is placed adjacent to each detector element (above the top layer and below the bottom layer). A thin plastic scintillator shield surrounds the D1 and D2 detector planes. We assume the measured values for detector energy resolution (@ $662 \mathrm{keV}$ in each detector) given above, and a trigger threshold of $20 \mathrm{keV}$ in both $\mathrm{D} 1$ and $\mathrm{D} 2$. We conservatively assume a ToF resolution of $400 \mathrm{ps}$ for the $\mathrm{LaBr}_{3} \mathrm{D} 2$. 


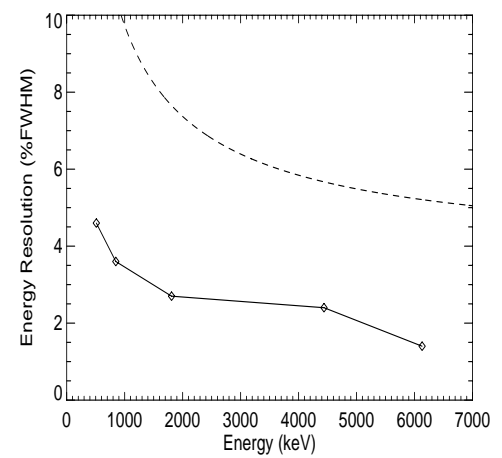

Figure 5: Energy resolution (\%FWHM) of the $\mathrm{LaBr}_{3}$ scintillator Compton telescope vs. energy. Dotted line indicates values for COMPTEL.

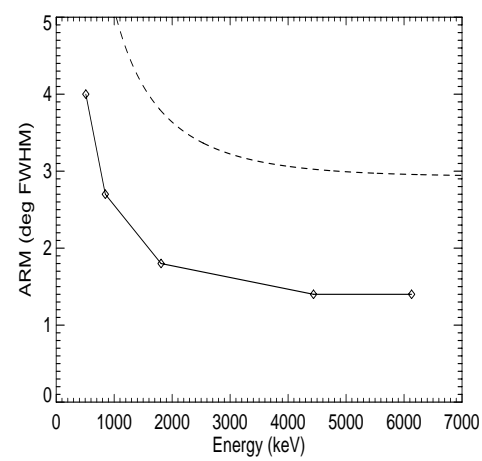

Figure 6: Angular resolution of the scintillator Compton telescope as measured by the ARM. Dotted line indicates values for COMPTEL.

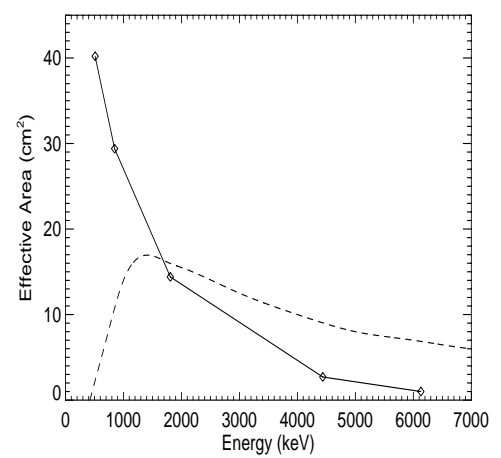

Figure 7: Effective area of the example scintillator Compton telescope for the data cuts given in the text. Dotted line indicates values for COMPTEL.

To estimate the sensitivity of an instrument based on the fast LaX scintillator technology described above, we have performed initial simulations of the response of a Compton telescope using the MGGPOD Monte Carlo package (Weidenspointner et al. 2005). We first simulated mono-energetic photons at five different energies $(511 \mathrm{keV}, 847 \mathrm{keV}$, $1809 \mathrm{keV}, 4438 \mathrm{keV}$, and $6130 \mathrm{keV}$ ) with normal incidence to predict the telescope response (energy resolution, angular resolution, and effective area $\left.A_{\text {eff }}\right)$. Simple data cuts were used: events were allowed if there was exactly one trigger in D1 and either one or two triggers in D2. In the case of two D2 triggers, the hit in the top D2 layer was assumed to be the first. The computed Compton scatter angle was required to be in the range 0-53 ${ }^{\circ}$. The results are plotted in Figs. 5-7. The dotted line indicates the values from COMPTEL calibration measurements (Schönfelder et al. 1993). The angular resolution is given in terms of the angular resolution measure (ARM), the difference between the computed and true scatter angles. The effective area is given for events falling within $3 \sigma$-wide windows around the incident energy, a ToF value of $2.5 \mathrm{~ns}$, and an ARM of $0^{\circ}$. A Compton telescope based on $\mathrm{LaBr}_{3}$ will significantly outperform COMPTEL in energy and angular resolution. Below $\sim 2 \mathrm{MeV}$ an equal or significantly larger effective area can be achieved. This is impressive performance for an instrument less than $25 \%$ the volume of COMPTEL. At higher energies the effective area of the proposed telescope will likely be improved by more sophisticated event reconstruction methods.

To estimate the magnitude of the background reduction we might expect, we examined a sample ToF spectrum from COMPTEL. We focus on the most difficult background range, that of 4.1-6 MeV. Although the absolute background rate is low compared to other bands, this range suffers from a continuum background with no spectral features to aid in its removal. Fig. 8 shows the COMPTEL ToF spectrum from 4.2-6 MeV used in the CDG analysis (Kappadath 1998). Since our proposed methods will have the greatest effect on activation background, we chose this energy interval because it sits above all the activation lines, thereby making it the most challenging energy range for improvement. However, it is important to note that more fertile ground for background rejection lies below this range, because of identifiable line signatures that remedial action that can be taken to reduce the buildup. Below $4.2 \mathrm{MeV}$, the background rejection techniques we discuss can be employed to make very large improvements. Because of the absence of radioactive contamination, the energy range from 4.2-6 MeV represented the best COMPTEL performance range. It falls above the intense contamination from ${ }^{24} \mathrm{Na},{ }^{22} \mathrm{Na},{ }^{28} \mathrm{Al}$, and neutron capture $(2.2 \mathrm{MeV})$. The primary background here comes from prompt neutron cascades in material in the aperture of the instrument (along with accidental coincidences and cascades from elsewhere in the instrument and spacecraft, which we will eliminate via ToF discrimination). The different background components are shown as fitted: Type A events (right Gaussian), Type C events near D1 (left Gaussian), Type B events (constant), and Type C \& D events from the rest of the instrument (exponential continuum). The Type A events with the proper ToF are $\gamma$ rays coming down the boresight of the instrument produced in the field of view by undetected neutrons. They constitute the most elusive form of background. We have conservatively estimated how these background components might rescale in a telescope with $\mathrm{LaBr}_{3}$ (Fig. 9) for D2 on the assumption that count rates scale with detector mass. We reduced the Gaussian widths to match the assumed ToF resolutions and those peaks were rescaled by the ratio of the total D1 mass to the COMPTEL D1 mass. The Type $\mathrm{C}$ events were further reduced by a factor of two to allow for the use of a material, such as Be, less subject to activation. The Type D continuum was scaled by the ratio of the overall telescope mass, which we conservatively assumed would scale as the ratio of the detector masses. The Type B events were scaled by the product of the D1 and D2 mass ratios. The number of counts within the valid ToF window (vertical dotted lines) was then compared to that of COMPTEL. The only significant remaining background is from Type A events. This simple estimate yielded roughly a 


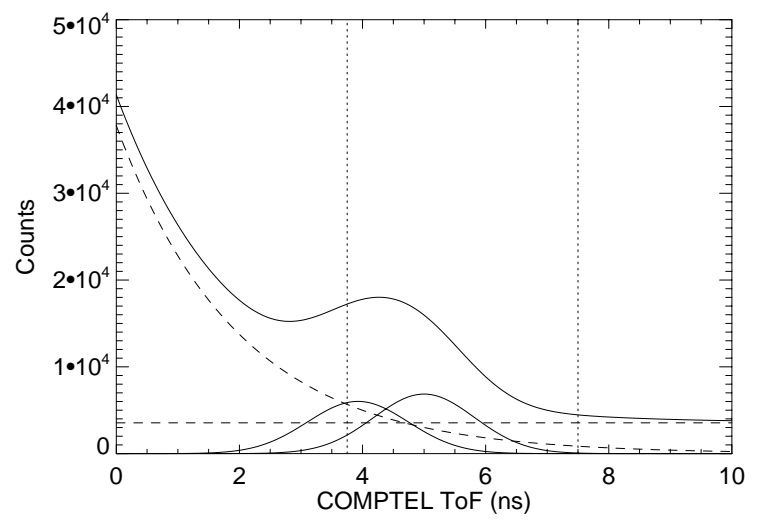

Figure 8: COMPTEL ToF spectrum from 4.2-6 MeV. Components are described in the text. Dotted vertical lines indicate ToF window for good events.

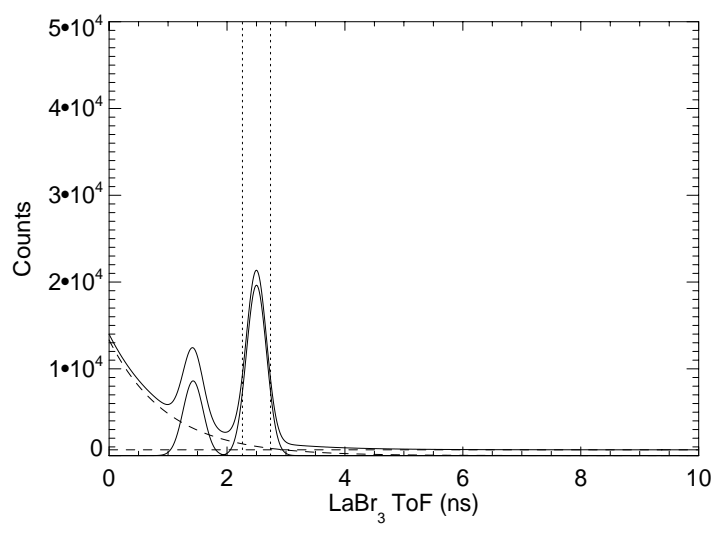

Figure 9: Estimated ToF spectrum of a Compton telescope using $\mathrm{LaBr}_{3}$ for D2. Components are scaled from Fig. 8 as described in the text.

factor of 2.5 reduction in background assuming that the amount of passive material in front of D1 remains unchanged in quantity and composition. This is the reduction expected for observations of isotropic, continuum sources such as the CDG. To see how this would affect the sensitivity for point-line sources, we scaled this background by the ratio of the permitted energy (Fig. 5) and ARM (Fig. 6) windows, as appropriate for observations of point-like, line sources (e.g., $\mathrm{SNe}$, SNR, knots of ${ }^{26} \mathrm{Al}$ or ${ }^{60} \mathrm{Fe}$ emission, etc.). This gives a rough estimate of the background reduction possible with the baseline Compton telescope model: a factor of $\sim 20$ for a $\mathrm{LaBr}_{3} \mathrm{D} 2$. Using the simulated effective areas we may also estimate the relative line sensitivity to a point source located on-axis. At $1809 \mathrm{keV}$, assuming the background scales as it does from 4.2-6 MeV (a conservative overestimate of the background), we expect to improve on the COMPTEL sensitivity (in units of $\sigma$ ) by a factor of $\sim 4\left(\mathrm{LaBr}_{3}\right)$.

This sensitivity estimate represents a "worst case" comparison and we expect to do much better in practice. The background reduction was estimated from best performance energy band of COMPTEL, whereas below $4 \mathrm{MeV}$ the reduction would be significantly greater due to the activation background that plagued COMPTEL. The largest background component remaining, the Type A events, arises from neutron interactions in passive material in the telescope aperture. This can be reduced by aggressively limiting this passive material. Additional reduction factors of $\sim$ several can be expected because of the absence of the neighboring EGRET and OSSE telescopes and the selection of a low-inclination orbit (the CGRO orbit was at $\sim 28^{\circ}$ ) that prevents SAA activation. Finally, we note that Type A events will be a significant, inescapable source of background in any Compton telescope design, and that liquid organic scintillator offers far fewer decay chains than higher- $Z$ detector materials. Given these considerations, we find the factor of $\sim 4$ improvement in sensitivity derived using the relatively clean range of 4-6 $\mathrm{MeV}$ encouraging. We estimate that the background problem is four times worse in the range of 2.7-4.2 MeV and about twice as bad from 1.5-2.7 MeV judging from the COMPTEL S/N ratio of background count rate to cosmic diffuse count rate. Thus, for objects that radiate in these ranges large improvements are possible using the techniques described above, especially if their spectrum is harder than the cosmic diffuse spectrum (e.g., lines). At the higher energies the segmentation of the detectors will improve the imaging properties of the detector, because both the mean free path of a Compton scattered photon and sometimes the range of the scattered electron will be comparable to or greater than the segmentation size. Knowing where the reactions were initiated to $2 \mathrm{~cm}$ will reduce the geometrical uncertainties in the imaging exercise. We have not estimated the magnitude of this advantage over the COMPTEL instrument at this point, but it will increase with energy.

The improved energy resolution, with respect to COMPTEL, is achieved with small detector volumes with large PMT coverage and the use of high-resolution scintillators such as $\mathrm{LaBr}_{3}$. The energy resolution is slightly degraded from pure $\mathrm{LaBr}_{3}$ because some energy is deposited in the liquid scintillator. Despite this, we have simulated the complex countrate spectrum from a solar flare and compared it to that recorded using a monolithic $3^{\prime \prime} \times 3^{\prime \prime}$ cylindrical NaI detector, the material used by both GRS on SMM and OSSE instruments (Fig. 10). Not only are the individual lines much narrower, but also after selecting events based on imaging information, the suppression of the Compton tails is quite significant (compare to the input spectrum, shown in the inset). This enables one to much more easily and unambiguously deconvolve the complex solar spectrum of narrow lines, broad lines and continuum. For example, this power could be applied to any Type Ia supernova spectrum measured, undoubtedly allowing one to rule out many conflicting models. Much SN progress can be made with $\sim 3 \%$ energy resolution at $847 \mathrm{keV}$, because the ${ }^{56} \mathrm{Ni}$ lines are separated by more 
than this $(\sim 4 \%)$ and they vary significantly in intensity depending on the model and the age of the SN. The lines themselves are Doppler broadened to the 3\% level.

\section{CONCLUSIONS}

The use of $\mathrm{LaX}$, specifically $\mathrm{LaBr}_{3}$, scintillators can enable Compton telescopes to make great improvements in sensitivity by

- Significantly improving the ToF resolution of the instrument, thereby, greatly reducing the rate of accidental or chance coincidences,

- Using the ToF resolution to reject background cascade $\gamma$ ray events from sites other than those in almost direct contact with the forward scatterer,

- Using the ToF resolution to move the two detector planes closer together to boost sensitive area, while maintaining a constant mass,

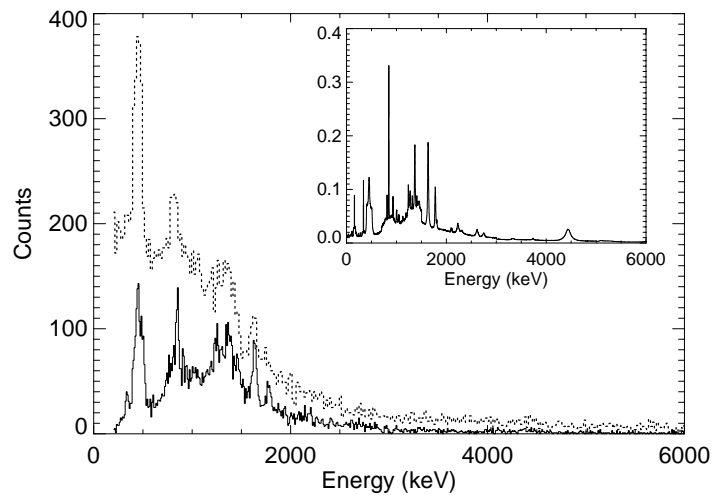

Figure 10: Simulated solar flare spectrum (inset) as measured by the $\mathrm{LaBr}_{3}$ Compton telescope (solid) and monolithic NaI detector (dotted).

- Significantly improving the energy resolution to isolate background line features,

- Using the superior energy resolution to improve sensitivity for narrow lines, such as from ${ }^{26} \mathrm{Al}$ in the Galactic plane and from solar flares,

- Using the better energy resolution to reduce scatter angle computation errors and thus improve angular resolution and sensitivity.

The adoption of new scintillator technology will allow significant improvements in Compton telescope sensitivity while still retaining the advantages of basic scintillation detection. This in turn will allow a new generation of Compton telescopes to survey the sky, perform new science and identifying more objects for further study with subsequent mission. All this can is enabled by the adoption of LaX scintillators with corresponding minor changes elsewhere in the instrument design.

\section{REFERENCES}

1. Bloemen, H., et al., 1999, The revised COMPTEL Orion results, Astrophysical Journal, 521, L137.

2. Diehl, R., et al., 1995, COMPTEL observations of Galactic ${ }^{26} \mathrm{Al}$ emission, Astronomy and Astrophysics, 298, 445.

3. Dingus, B.L., 1995, EGRET Observations of $>30 \mathrm{MeV}$ Emission from the Brightest Bursts Detected by BATSE, Astrophysics and Space Science, 231, 187.

4. Iyudin, A.F., et al., 1994, COMPTEL observations of Ti-44 gamma-ray line emission from CAS A, Astronomy and Astrophysics, 284, L1.

5. Kappadath, S. C., 1998, Measurement of the cosmic diffuse gamma-ray spectrum from $800 \mathrm{keV}$ to $30 \mathrm{MeV}$, Ph.D. Thesis, University of New Hampshire.

6. Kuhn, A., et al., 2004, Design of a Lanthanum Bromide Detector for Time-of-Flight PET, IEEE Transactions on Nuclear Science, 51, 2550.

7. McConnell, M.L., et al., 2002, The Soft Gamma-Ray Spectral Variability of Cygnus X-1, Astrophysical Journal, 572, 984 .

8. McConnell, M. L., \& Ryan, J. M., 2004, Status and prospects for polarimetry in high energy astrophysics, New Astronomy Reviews, 48, 215.

9. Morris, D. J., et al., 1998, Reassessment of the ${ }^{56}$ Co emission from SN 1991T, Proceedings of the Fourth Compton Symposium (AIP 410), 1084.

10. Ryan, J. M., 1978, Energy and angle distributions for atmospheric and cosmic diffuse gamma rays from 2 to 25 $\mathrm{MeV}, \mathrm{Ph} . \mathrm{D}$. Thesis, University of California, Riverside.

11. Ryan, J. M., 2004, Astrophysics challenges of MeV astronomy instrumentation, New Astronomy Reviews, 48, 199. 
12. Schönfelder, V., et al., 1977, Diffuse cosmic and atmospheric MeV gamma radiation from balloon observations, Astrophysical Journal, 217, 306.

13. Schönfelder, V., et al., 1993, Instrument description and performance of the imaging gamma-ray telescope COMPTEL aboard the Compton Gamma-Ray Observatory, Astrophysical Journal Supplement Series, 86, 657.

14. Shah, K. S., et al., 2003, $\mathrm{LaBr}_{3}$ :Ce Scintillators for Gamma Ray Spectroscopy, IEEE Transactions on Nuclear Science, 50, 2410.

15. Share, G. H., et al., 1988, SMM detection of diffuse galactic $511 \mathrm{keV}$ annihilation radiation, Astrophysical Journal, 326, 717.

16. Strong, A., et al., 2000, Diffuse continuum gamma rays from the galaxy, Astrophysical Journal, 537, 763.

17. Weidenspointner, G., 1999, The origin of the cosmic gamma-ray background in the COMPTEL energy range, Ph.D. Thesis, Technical University Munich, Germany.

18. Weidenspointner, G., et al., 2001, The COMPTEL instrumental line background, Astronomy \& Astrophysics, $\mathbf{3 6 8}$, 347.

19. Weidenspointner, G., et al., 2005, MGGPOD: a Monte Carlo Suite for Modeling Instrumental Line and Continuum Backgrounds in Gamma-Ray Astronomy, Astrophysical Journal Supplement Series, 156, 69.

20. White, R. S., et al., 1977, Cosmic diffuse gamma rays from 2 to $25 \mathrm{MeV}$, Astrophysical Journal, 218, 920. 